\title{
Francois H. Cornet: Elements of crustal geomechanics
}

\author{
Cambridge University Press, 2015, ISBN 978-0-521-87578-3
}

\author{
Derek Elsworth • Yves Guglielmi
}

Received: 2 November 2015/ Accepted: 3 November 2015/Published online: 11 November 2015

(C) Springer International Publishing Switzerland 2015

This new book by Cornet is a refreshing addition to the variety of books on geomechanics in that it attempts to straddle both geo-engineering and geo-science with their inherently different length and timescales. In that regard, the book melds a traditional text on rock mechanics-discussing all the components of strain and stress implicit in elasticity-but then including models for both brittle and ductile failure, important as the crust is traversed. Usefully, the book then introduces concepts of fluid transport from the same perspective of the conservations equations of mass and momentum and appropriate constitutive laws already used for the solid phase. This presentation inherently demonstrates the equivalence of the mechanics of solids and fluids in their formalism before introducing macroscopic equations for equivalent media-again, both for solids and fluids. As such, the book provides significant clarity of the equivalence of these approaches in a straightforward way but still finishes with the more usual representations of these approaches as used in a continuum sense. The presentation of these concepts is well executed

\section{Elsworth $(\bowtie)$}

Pennsylvania State University, University Park, PA, USA

e-mail: elsworth@psu.edu

\section{Y. Guglielmi}

University of Aix-Marseille, Marseille, France

e-mail: guglielmi@cerege.fr and clear-and in this particular assemblage in the presentation is unique among texts. This mixture of mechanics and geofluids, so presented, would be unique in a book by itself. However, the text continues with an extension to fault constitutive equations and an exposition of dynamic elasticity in application to the key concepts of crustal seismology discussed from the perspective of fault mechanics. Of course, this is the appropriate method to link the key issues of failure and solid mechanics discussed previously and this is followed with a discussion of the roles of fluids in triggering such failure. The text concludes with a definition of methods of field experimentation then illustrated with the results of characteristic site investigations in both igneous and sedimentary sites/ basins to illustrate the importance of the prior discussions in describing key processes that operate in these different environments. In ensemble, the book provides an interesting and unique perspective of stress measurements in faulted and fractured environments-of which the author has been a significant contributor to the contemporary science literature.

The book covers a significant landscape-elasticity, momentum-based fluid flows, brittle-throughductile mechanisms of deformation and their homogenized form, the pseudo-static and dynamic response of faults, poromechanics, field experimentation and the application of these principles to describe crustal rheology. This is a significant undertaking - at some times the presentation seems staccato because of the 
broad delivery-however, in general the topics hold together well and the text flows smoothly.

This book provides an excellent theoretical treatment of contemporary geomechanics — solid, fluid and thermal interactions in rocks accommodating rheology and rupture for static through dynamic conditions. Although presented at a high-level, concepts are eminently accessible and aided by examples to highlight relevance in both geophysics and in engineering. Indeed, many examples taken from experiments conducted by the author include original data. This is destined to become a standard reference text for serious scholars spanning pure and applied geophysics through engineering geomechanics with application to a process-based understanding in natural and engineered systems. 